Preprint typeset in JHEP style - HYPER VERSION

UUITP-13-03

YITP-SB-03-38

hep-th/0309015

\title{
Time dependent solitons of noncommutative Chern-Simons theory coupled to scalar fields
}

\author{
Leszek Hadasz* \\ Laboratoire de Physique Thèorique, \\ Univ. Paris-Sud, Bât. 210, F-91405 Orsay Cedex \\ hadasz@th.u-psud.fr \\ UIf Lindström \\ Department of Theoretical Physics, \\ Uppsala University, Box 803, SE-751 08 Uppsala, Sweden \\ ulf.lindstrom@teorfys.uu.se \\ Martin Roček \\ State University of New York \\ Stony Brook, NY 11794-3840, USA \\ rocek@insti.physics.sunysb.edu

\section{Rikard von Unge} \\ Institute for Theoretical Physics and Astrophysics \\ Faculty of Science, Masaryk University \\ Kotlárská 2, CZ-611 37, Brno, Czech Republic \\ unge@physics.muni.cz
}

AbStract: We study one- and two-soliton solutions of noncommutative Chern-Simons theory coupled to a nonrelativistic or a relativistic scalar field. In the nonrelativistic case, we find a tower of new stationary time-dependent solutions, all with the same charge density, but with increasing energies. The dynamics of these solitons cannot be studied using traditional moduli space techniques, but we do find a nontrivial symplectic form on the phase space indicating that the moduli space is not flat. In the relativistic case we find the metric on the two soliton moduli space.

KEYwORDS: noncommutative solitons, Chern-Simons theory.

\footnotetext{
* M. Smoluchowski Institute of Physics, Jagiellonian University, Reymonta 4, 30-059 Kraków, Poland
} 


\section{Contents}

1. Introduction 1

2. Pure Chern-Simons 2

3. The nonrelativistic scalar field 3

3.1 The one soliton case 1

3.1.1 The solution

3.1.2 The shape of the one soliton solution

3.1.3 More stationary solutions 6

3.1.4 Moving one-soliton solutions

3.2 The two soliton case 9

3.2.1 The solution 9

3.2.2 The shape of the two soliton solution 10

3.2 .3 The dynamics

4. The relativistic scalar field 14

4.1 The one soliton case 16

4.1.1 The static solution 16

4.1.2 More stationary solutions 16

4.2 The two soliton case $\overline{16}$

4.2 .1 The solution 16

4.2 .2 The dynamics 17

5. Conclusions 19

\section{Introduction}

Soliton solutions of noncommutative field theory (for an introduction to the subject and references see for instance [1, 2, 3, 4) is a fascinating subject. In some cases, theories that do not have soliton solutions classically turn out to have them when made noncommutative [5]. The fact that these solitons have an interpretation as lower dimensional D-branes in the context of string-theory [6, 1] and as quasi-particles in the context of noncommutative descriptions of solid state systems [7, 8] makes this phenomenon even more interesting. We therefore believe that it is worthwhile to study solitonic configurations and their detailed dynamics for a range of noncommutative theories [9, 10].

Noncommutative Chern-Simons theory is particularly interesting, as Susskind has argued that it describes the fractional quantum Hall effect [7], (see also [8, 11, 12, 13, 14]). 
In Susskind's description the solitons are the quasiparticles that exhibit fractional statistics and are responsible for the unusual quantum behavior of the QHE system. According to Susskind, it is the noncommutativity of the theory that encodes the graininess of the two-dimensional charged fluid in a magnetic field.

In this work we study soliton solutions of noncommutative Chern-Simons theory coupled to relativistic and nonrelativistic scalar fields [15, 16]; these theories are noncommutative versions of the Jackiw-Pi model [17, 18]. We find the shape of two-soliton configuration using the exact Seiberg-Witten map [19, 20, 21, 22]. We also find new stationary timedependent single soliton solutions, all with the same $\delta$-function charge density profile, but with increasing energies. Finally, in the relativistic case, we are able to find the dynamics of the two soliton solution using traditional moduli space techniques.

We think that our results are novel and striking; however, in contrast to the case of pure noncommutative Chern-Simons theory, the details of the physical interpretation, in particular the relation to the fractional quantum Hall effect, remain to be sorted out.

This paper is a thoroughly revised version. We thank P. A. Horvathy, L. Martina and P. C. Stichel for illuminating comments that led us to reconsider some of our previously reported results; see note added at the end of the paper.

\section{Pure Chern-Simons}

We take our noncommutative space to be defined by the commutation relation $[\hat{x}, \hat{y}]=-i \theta$. Noncommutative Chern-Simons theory is defined by the Lagrangian

$$
L=-\pi \kappa \theta \epsilon^{\alpha \beta \gamma} \operatorname{Tr}\left(A_{\alpha} \partial_{\beta} A_{\gamma}-\frac{2 i}{3} A_{\alpha} A_{\beta} A_{\gamma}\right),
$$

where $\kappa$ is the Chern-Simons level, representing the (inverse) filling fraction in the application to the fractional quantum Hall effect ${ }^{1}$. Introducing the notation $X^{i}=x^{i}-\theta \epsilon_{i j} A_{j}$ we write the Lagrangian as [23, 24, 25]

$$
L=-\frac{\pi \kappa}{\theta} \operatorname{Tr}\left(-\epsilon_{i j} X^{i}\left(\dot{X}^{j}-i\left[A_{0}, X^{j}\right]\right)+2 \theta A_{0}\right) .
$$

We may introduce the complex notation

$$
\begin{aligned}
c & =\frac{1}{\sqrt{2 \theta}}\left(x^{1}-i x^{2}\right), \\
K & =\frac{1}{\sqrt{2 \theta}}\left(X^{1}-i X^{2}\right),
\end{aligned}
$$

in which the Lagrangian can be written 15

$$
L=i \pi \kappa \operatorname{Tr}\left(K^{\dagger} D_{t} K-K D_{t} K^{\dagger}\right)-2 \pi \kappa \operatorname{Tr} A_{0},
$$

where we have also introduced $D_{t} K=\partial_{t} K-i\left[A_{0}, K\right]$. The equations of motion are

$$
\begin{aligned}
{\left[K, K^{\dagger}\right] } & =1, \\
D_{t} K & =0 .
\end{aligned}
$$

\footnotetext{
${ }^{1}$ In what follows we shall need to take $\kappa$ positive to find our solutions.
} 
Susskind argues [7] that in the presence of a quasiparticle the first equation of (2.5) gets modified to

$$
\left[K, K^{\dagger}\right]=1+q|0\rangle\langle 0|
$$

where $|q|<1$. In [26] this system was studied from the point of view of String Theory. The shape of the solutions was found using the exact Seiberg-Witten map [19, 20, 21, 22] and it was shown that the solution represents a deficit or excess of charge centered around the origin. However, this solution does not have any moduli, which we need to study moving solutions. The situation improves when we include a scalar field, which has been argued to describe density fluctuations of the quantum Hall liquid [15]. In this case the solutions have moduli describing the positions of the independent solitons, so we should be able to make them move around.

\section{The nonrelativistic scalar field}

The Lagrangian for noncommutative Chern-Simons theory coupled to a nonrelativistic scalar field can be written as 15, 17]

$$
L=L_{C S}+2 \pi \theta \operatorname{Tr}\left(i D_{t} \phi \phi^{\dagger}-\frac{1}{2 m} D_{i} \phi\left(D_{i} \phi\right)^{\dagger}+\lambda\left(\phi \phi^{\dagger}\right)^{2}\right)
$$

where $m$ is a mass parameter of dimension (length) $)^{-1}$ and $\lambda$ is a parameter of dimension length. This theory has a BPS bound in the case where $2 m \lambda \kappa=1$. Assuming this relation, rescaling the time variable $t \rightarrow m t$ and introducing the complex notation

$$
D=\sqrt{\frac{\theta}{2}}\left(D_{1}-i D_{2}\right)
$$

we may write the action in operator notation $a^{2}$

$$
\begin{aligned}
L= & i \pi \kappa \operatorname{Tr}\left\{K^{\dagger} D_{t} K-K D_{t} K^{\dagger}\right\}-2 \pi \kappa \operatorname{Tr} A_{0} \\
& +2 \pi \theta \operatorname{Tr}\left\{i D_{t} \phi \phi^{\dagger}-\frac{1}{2 \theta}\left(D \phi(D \phi)^{\dagger}+\bar{D} \phi(\bar{D} \phi)^{\dagger}\right)+\frac{1}{2 \kappa}\left(\phi \phi^{\dagger}\right)^{2}\right\},
\end{aligned}
$$

where $D, \bar{D}$ and $D_{t}$ are defined as

$$
\begin{aligned}
D_{t} \phi & =\dot{\phi}-i A_{0} \phi, \\
D \phi & =(K \phi-\phi c), \\
\bar{D} \phi & =-\left(K^{\dagger} \phi-\phi c^{\dagger}\right) .
\end{aligned}
$$

The action (3.3) is gauge invariant under the transformations

$$
\phi \rightarrow U \phi, K \rightarrow U K U^{\dagger}, \quad A_{0} \rightarrow U\left(i \partial_{t}+A_{0}\right) U^{\dagger},
$$

\footnotetext{
${ }^{2}$ In fact, a further rescaling is possible which leaves an action with the only free parameter an overall factor of $\kappa ; t \rightarrow \theta t, \phi \rightarrow \sqrt{\kappa / \theta} \phi, A_{0} \rightarrow A_{0} /(m \theta)$.
} 
where $U$ is an arbitrary unitary operator. The action is also invariant under translations by a constant $z$ :

$$
\phi \rightarrow \phi U(z), \quad K \rightarrow K+z, \quad A_{0} \rightarrow A_{0} \quad ; \quad U(z) \equiv e^{z c^{\dagger}-\bar{z} c}
$$

Extremizing the action (3.3) gives the equations of motion

$$
\begin{aligned}
i D_{t} \phi+\frac{1}{2 \theta}\{D, \bar{D}\} \phi+\frac{1}{\kappa} \phi \phi^{\dagger} \phi & =0, \\
1-\left[K, K^{\dagger}\right] & =\frac{\theta}{\kappa} \phi \phi^{\dagger}, \\
\left\{K, \phi \phi^{\dagger}\right\}-2 \phi c \phi^{\dagger} & =2 \kappa i D_{t} K .
\end{aligned}
$$

In the time-independent case one can show that any solution to the BPS equations

$$
\begin{aligned}
K^{\dagger} \phi-\phi c^{\dagger} & =0, \\
1-\left[K, K^{\dagger}\right] & =\frac{\theta}{\kappa} \phi \phi^{\dagger}, \\
A_{0} & =-\frac{1}{2 \kappa} \phi \phi^{\dagger},
\end{aligned}
$$

is also a solution to the full equations of motion.

\subsection{The one soliton case}

\subsubsection{The solution}

The static one-soliton solution discussed in [15] looks like

$$
\begin{aligned}
\phi & =\sqrt{\frac{\kappa}{\theta}}|0\rangle\langle z|, \\
K & =z|0\rangle\langle 0|+S_{1} c S_{1}^{\dagger}, \\
A_{0} & =-\frac{1}{2 \theta}|0\rangle\langle 0|,
\end{aligned}
$$

where we have introduced the shift operators $S_{n}=\sum_{i=0}^{\infty}|i+n\rangle\langle i|$ and the usual coherent state $|z\rangle=U(z)|0\rangle$. This solution can be found from the simple soliton at $z=0$ by a combination of the translation (3.6) and a gauge transformation with a unitary operator

$$
T(z)=e^{z K^{\dagger}-\bar{z} K} .
$$

\subsubsection{The shape of the one soliton solution}

To find the shape of the soliton solution we have to extract it from the operators $X_{1}$ and $X_{2}$, or equivalently from $K=\frac{1}{\sqrt{2 \theta}}\left(X^{1}-i X^{2}\right)$. This we do by using the exact SeibergWitten map [19, 20, 21, 22], which implies that the Fourier transform of the density is given by

$$
\tilde{\rho}(\mathbf{k})=\operatorname{Tr}\left(e^{-i k_{a} X^{a}}\right)=\operatorname{Tr}\left(e^{-i\left(\bar{k} K+k K^{\dagger}\right)}\right)
$$


where we have defined $k=\sqrt{\frac{\theta}{2}}\left(k_{1}-i k_{2}\right)$. We then have

$$
\rho(\mathbf{x})=\int \frac{d^{2} \mathbf{k}}{(2 \pi)^{2}} \tilde{\rho}(\mathbf{k}) e^{i k_{a} x^{a}}
$$

where $x^{a}$ are ordinary commuting coordinates. This form of the density is very natural if one thinks of the coordinates of the individual electrons as the eigenvalues of the $X$ matrices, since then $\tilde{\rho}=\sum_{i=1}^{N} e^{-i \mathbf{k} \cdot \mathbf{x}_{i}}$ is exactly the Fourier transform of the density $\rho=$ $\sum_{i=1}^{N} \delta\left(\mathbf{x}-\mathbf{x}_{i}\right)$.

To evaluate the Fourier moments we first notice that $K$ is composed of two pieces which commute with each other. This makes it possible to write

$$
\operatorname{Tr}\left(e^{-i\left(\bar{k} K+k K^{\dagger}\right)}\right)=\operatorname{tr}\left(e^{-i(\bar{k} z+k \bar{z})|0\rangle\langle 0|}\right)+\operatorname{Tr}^{\prime}\left(e^{-i\left(\bar{k} S_{1} c S_{1}^{\dagger}+k S_{1} c^{\dagger} S_{1}^{\dagger}\right)}\right)
$$

where we have introduced the notation tr for traces over a finite set of states (in this case over the set $|0\rangle$ ) and $\operatorname{Tr}^{\prime}$ for an infinite trace with some states omitted (in this case $|0\rangle$ is omitted). These traces can be evaluated separately. The first one is

$$
\left\langle 0\left|\left(e^{-i(\bar{k} z+k \bar{z})|0\rangle\langle 0|}\right)\right| 0\right\rangle=e^{-i(\bar{k} z+k \bar{z})}
$$

and the second one is

$$
\sum_{n=1}^{\infty}\left\langle n\left|\left(e^{-i\left(\bar{k} S_{1} c S_{1}^{\dagger}+k S_{1} c^{\dagger} S_{1}^{\dagger}\right)}\right)\right| n\right\rangle=\sum_{n=1}^{\infty}\left\langle n\left|\left(S_{1} e^{-i\left(\bar{k} c+k c^{\dagger}\right)} S_{1}^{\dagger}+1-S_{1} S_{1}^{\dagger}\right)\right| n\right\rangle
$$

Noticing that $S_{1}^{\dagger}$ will shift the state $|n\rangle$ to $|n-1\rangle$ and that $1-S_{1} S_{1}^{\dagger}$ is the projection operator on the space spanned by $|0\rangle$, we get

$$
\operatorname{Tr}^{\prime}\left(e^{-i\left(\bar{k} S_{1} c S_{1}^{\dagger}+k S_{1} c^{\dagger} S_{1}^{\dagger}\right)}\right)=\operatorname{Tr}\left(e^{-i\left(\bar{k} c+k c^{\dagger}\right)}\right)
$$

This trace we perform using coherent states

$$
\begin{aligned}
\operatorname{Tr}\left(e^{-i\left(\bar{k} c+k c^{\dagger}\right)}\right) & =\int \frac{d^{2} \mathrm{w}}{\pi}\left\langle w\left|e^{-i\left(\bar{k} c+k c^{\dagger}\right)}\right| w\right\rangle \\
& =\int \frac{d^{2} \mathrm{w}}{\pi}\left\langle w\left|e^{-i k c^{\dagger}} e^{-i \bar{k} c}\right| w\right\rangle e^{-\frac{k \bar{k}}{2}} \\
& =\int \frac{d^{2} \mathrm{w}}{\pi} e^{-i(k \overline{\mathrm{w}}+\bar{k} \mathrm{w})} e^{-\frac{k \bar{k}}{2}} \\
& =\frac{2 \pi}{\theta} \delta^{(2)}(\mathbf{k}) .
\end{aligned}
$$

Thus we find the result

$$
\operatorname{Tr}\left(e^{-i\left(\bar{k} K+k K^{\dagger}\right)}\right)=\frac{2 \pi}{\theta} \delta^{(2)}(\mathbf{k})+e^{-i(\bar{k} z+k \bar{z})} .
$$

Fourier transforming back we get

$$
\rho(\mathbf{x})=\frac{1}{2 \pi \theta}+\delta\left(x_{1}-\sqrt{2 \theta} \mathcal{R} e(z)\right) \delta\left(x_{2}-\sqrt{2 \theta} \mathcal{I} m(z)\right),
$$

which is clearly to be interpreted as a constant background density $\frac{1}{2 \pi \theta}$ with a delta function type deformation ${ }^{3}$ located at $\sqrt{2 \theta} z$.

${ }^{3}$ Note that the sign of the deformation is opposite to that of the quasiparticles studied by Susskind 7$]$. 


\subsubsection{More stationary solutions}

The configuration (3.9) turns out to be a member of a huge family of stationary solitons

with a time independent density of the form (3.19). To see this consider an Ansatz

$$
\phi=\sqrt{\frac{\kappa}{\theta}}|0\rangle\left\langle\psi\left|, \quad A_{0}=-\frac{1}{2 \theta}\right| 0\right\rangle\langle 0|, \quad K=S_{1} c S_{1}^{\dagger}
$$

Inserting (3.20) into the equations of motion (3.7) we get

$$
\begin{aligned}
\langle\psi \mid \psi\rangle & =1, \\
\langle\psi|c| \psi\rangle & =0, \\
-i \partial_{\tau}|\psi\rangle & =c^{\dagger} c|\psi\rangle,
\end{aligned}
$$

where $\tau=\frac{t}{\theta}$.

The general solution of (3.23) reads

$$
|\psi\rangle=\sum_{n=0}^{\infty} \frac{b_{n} \mathrm{e}^{i n \tau}}{\sqrt{n !}}|n\rangle
$$

where $b_{n}$ are time-independent and satisfy

$$
\begin{gathered}
\sum_{n=0}^{\infty} \frac{\left|b_{n}\right|^{2}}{n !}=1, \\
\sum_{n=0}^{\infty} \frac{\overline{b_{n}} b_{n+1}}{n !}=0 .
\end{gathered}
$$

If we introduce the generating function

$$
G(\zeta)=\sum_{n=0}^{\infty} \frac{b_{n}}{n !} \zeta^{n}
$$

then $(3.25)$ and $(3.26)$ can be rewritten as

$$
\begin{aligned}
\frac{1}{\pi} \int_{\mathbb{C}} d^{2} \zeta \overline{G(\zeta)} G(\zeta) \mathrm{e}^{-\zeta \bar{\zeta}} & =1, \\
\frac{1}{\pi} \int_{\mathbb{C}} d^{2} \zeta \overline{\zeta G(\zeta)} G(\zeta) \mathrm{e}^{-\zeta \bar{\zeta}} & =0 .
\end{aligned}
$$

Any function $G$ which satisfies these relations yields a solution to (3.7).

We may think of the states $\left|\psi_{n}\right\rangle \equiv e^{i n \tau}|n\rangle$ as a basis and the combination (3.24) as the expression for a general state in this basis. The energy of a configuration can be computed from

$$
E=\pi \operatorname{Tr}\left(D \phi(D \phi)^{\dagger}+\bar{D} \phi(\bar{D} \phi)^{\dagger}-\frac{\theta}{\kappa}\left(\phi \phi^{\dagger}\right)^{2}\right)
$$


which for our particular ansatz becomes

$$
E=\frac{\pi \kappa}{\theta}\left(\left\langle\psi\left|c c^{\dagger}\right| \psi\right\rangle+\left\langle\psi\left|c^{\dagger} c\right| \psi\right\rangle-\langle\psi \mid \psi\rangle\right)=\frac{2 \pi \kappa}{\theta}\left\langle\psi\left|c^{\dagger} c\right| \psi\right\rangle .
$$

The "basis" states thus have energy

$$
E_{n}=\frac{2 \pi \kappa}{\theta} n
$$

and a general state has energy

$$
E=\frac{2 \pi \kappa}{\theta} \sum_{n=1}^{\infty} \frac{\left|b_{n}\right|^{2}}{(n-1) !}=\frac{2 \kappa}{\theta} \int_{\mathbb{C}} d^{2} \zeta|\zeta G(\zeta)|^{2} \mathrm{e}^{-\zeta \bar{\zeta}}
$$

which can be arbitrary.

More general solutions can be found by translating these solitons to arbitrary $z$ using the transformations (3.6); a simpler form of the translated solution can be found by combining the translation with the gauge transformation (3.10). The naive energy density is not gauge invariant; the physical energy density is found by performing a Seiberg-Witten map (3.11). We find that the physical energy density is translated and is concentrated at the position of the soliton.

The basis states $\left|\psi_{n}\right\rangle$ can be distinguished from more general configurations in a gauge invariant way. If we consider invariants of the form

$$
I[f]=\operatorname{Tr}\left(\phi^{\dagger} \phi f\left(c, c^{\dagger}\right)\right),
$$

then for generic functions $f$, we find that $I[f]$ is time independent only for the basis states. The original soliton (3.9) is the only solution to the BPS equations (3.8) in the family constructed here.

\subsubsection{Moving one-soliton solutions}

To make the solitons move one may use the "exotic" Galilean invariance of the theory. The infinitesimal version of this tranformation was found in [27]. Defining an infinitesimal complex paramteter $v=\frac{1}{\sqrt{2 \theta}}\left(v_{1}-i v_{2}\right)$ and writing everything in the operator notation, the transformations look like

$$
\begin{aligned}
\delta \phi & =t\left[v c^{\dagger}-\bar{v} c, \phi\right]+i \theta \phi\left(v c^{\dagger}+\bar{v} c\right), \\
\delta K & =t\left[v c^{\dagger}-\bar{v} c, K\right]+v t \\
\delta A_{0} & =t\left[v c^{\dagger}-\bar{v} c, A_{0}\right]+i\left(v\left(K^{\dagger}-c^{\dagger}\right)-\bar{v}(K-c)\right) .
\end{aligned}
$$

This transformation can be integrated to finite values of the parameter $v$. The transformation becomes

$$
\begin{aligned}
\phi & \rightarrow U \phi V^{\dagger} \\
K & \rightarrow U(K+v t) U^{\dagger} \\
A_{0} & \rightarrow U\left(A_{0}+i\left(v\left(K^{\dagger}-c^{\dagger}\right)-\bar{v}(K-c)\right)\right) U^{\dagger}
\end{aligned}
$$


which can be checked to leave the equations of motion invariant. Here $U$ is the unitary translation operator which translates a state by $v t$

$$
U(v t)=e^{\left(v c^{\dagger}-\bar{v} c\right) t}
$$

and $V$ is also a unitary translation operator, but it translates by $v(t-i \theta)$. It is given by

$$
V(v t)=e^{v(t-i \theta) c^{\dagger}-\bar{v}(t+i \theta) c} .
$$

The transformations (3.35) close under group multiplication up to a $\mathrm{U}(1)$ gauge transformation:

$$
U\left(v_{1} t\right) U\left(v_{2} t\right) \phi V^{\dagger}\left(v_{2} t\right) V^{\dagger}\left(v_{1} t\right)=e^{\frac{1}{2} \theta^{2}\left(v_{2} \bar{v}_{1}-v_{1} \bar{v}_{2}\right)} U\left(\left(v_{1}+v_{2}\right) t\right) \phi V^{\dagger}\left(\left(v_{1}+v_{2}\right) t\right)
$$

as the $\mathrm{U}(1)$ transformation is constant and commutative, it does not act on $K$ and $A_{0}$.

The form of the Galilean tranformation can be improved by acting with an additional gauge transformation (3.5). In particular, choosing the unitary operator $U^{\dagger}(v t)$, one can write the action of the Galilean transformation as

$$
\begin{aligned}
\phi & \rightarrow \phi V^{\dagger} \\
K & \rightarrow K+v t \\
A_{0} & \rightarrow A_{0}+i\left(v K^{\dagger}-\bar{v} K\right) .
\end{aligned}
$$

Note the similarity to the translations (3.6).

When $K$ is time-independent, the shift in $A_{0}$ can then be eliminated by a further gauge transformation with

$$
T=e^{t v K^{\dagger}-t \bar{v} K}
$$

giving us the final galilean transformation

$$
\begin{aligned}
\phi & \rightarrow T \phi V^{\dagger}, \\
K & \rightarrow T K T^{\dagger}+v t, \\
A_{0} & \rightarrow T A_{0} T^{\dagger}
\end{aligned}
$$

we emphasize that the simple homogeneous transformation of $A_{0}$ requires time-independent $K$.

As an example, the $z_{0}=0$ static soliton

$$
\begin{aligned}
\phi & =\sqrt{\frac{\kappa}{\theta}}|0\rangle\langle 0|, \\
K & =S_{1} c S_{1}^{\dagger}, \\
A_{0} & =-\frac{1}{2 \theta}|0\rangle\langle 0|,
\end{aligned}
$$


can be boosted using (3.41), as $K$ is time-independent. Here, the operator $T$ is block diagonal and acts trivially on $|0\rangle$. Also, one may show that $T K T^{\dagger}=K-v t\left[K, K^{\dagger}\right]$. Thus we find the boosted operators

$$
\begin{aligned}
\phi & =\sqrt{\frac{\kappa}{\theta}}|0\rangle\langle v(t-i \theta)|, \\
K & =v t|0\rangle\langle 0|+S_{1} c S_{1}^{\dagger}, \\
A_{0} & =-\frac{1}{2 \theta}|0\rangle\langle 0|,
\end{aligned}
$$

where $|v(t-i \theta)\rangle$ is the coherent state at $z=v(t-i \theta)$. Other solutions can be generated by boosting more general stationary solutions, e.g., solutions centered at some point $z_{0}$ or the new stationary solutions described above.

\subsection{The two soliton case}

\subsubsection{The solution}

After performing the gauge transformation (3.5) with $U=|0\rangle\langle 1|+| 1\rangle\langle 0|+S_{2} S_{2}^{\dagger}$ the two soliton solution found in 15 can be written as

$$
\begin{aligned}
\phi & =\sqrt{\frac{2 \kappa}{\theta}}(A|0\rangle\langle+|+B| 1\rangle\langle-|) \\
K & =z\left(C|0\rangle\left\langle 1\left|+\frac{1}{C}\right| 1\right\rangle\langle 0|\right)+S_{2} c S_{2}^{\dagger},
\end{aligned}
$$

where $| \pm\rangle$ are the normalized states

$$
| \pm\rangle=\frac{1}{\sqrt{2\left(1 \pm \mathrm{e}^{-2|z|^{2}}\right)}}(|z\rangle \pm|-z\rangle), \quad|z\rangle=\mathrm{e}^{z c^{\dagger}-\bar{z} c}|0\rangle
$$

The BPS equations now tell us that $|A|^{2}+|B|^{2}=1$, which is solved by $A=\sin (\alpha)$ and $B=\cos (\alpha)$, that $C=\cot (\alpha) \sqrt{\operatorname{coth}|z|^{2}}$, and that $\alpha$ has to satisfy the equation

$$
|z|^{2}\left(C^{2}-\frac{1}{C^{2}}\right)=B^{2}-A^{2}
$$

This is a cubic equation in $\tan \alpha$, so it can be solved explicitly. The solution is not particularly illuminating, so we explicitly give only some limiting properties and use the full result in the calculations. We find that

$$
\begin{aligned}
& C=1-\frac{e^{-2|z|^{2}}}{4|z|^{2}-1}+\mathcal{O}\left(e^{-4|z|^{2}}\right) \quad|z| \gg 1 \\
& C=|z|\left(1+\frac{1}{2}|z|^{4}-\frac{11}{24}|z|^{8}+\mathcal{O}\left(|z|^{12}\right)\right) \quad|z| \ll 1 .
\end{aligned}
$$




\subsubsection{The shape of the two soliton solution}

Using the same method as in the one soliton case we can calculate the shape of the two soliton solution. The Fourier moments of the density are given by

$$
\tilde{\rho}(\mathbf{k})=\operatorname{Tr}\left(e^{-i\left(\bar{k} K+k K^{\dagger}\right)}\right)
$$

which again splits into two pieces

$$
\operatorname{Tr}\left(e^{-i\left(\bar{k} K+k K^{\dagger}\right)}\right)=\operatorname{tr}\left(e^{-i(D|0\rangle\langle 1|+\bar{D}| 1\rangle\langle 0|)}\right)+\operatorname{Tr}^{\prime}\left(e^{-i\left(\bar{k} S_{2} c S_{2}^{\dagger}+k S_{2} c^{\dagger} S_{2}^{\dagger}\right)}\right)
$$

where we have introduced $D=\bar{k} z C+\frac{k \bar{z}}{C}$ and where tr now means the trace over the subspace spanned by $|0\rangle,|1\rangle$ and $\operatorname{Tr}^{\prime}$ means the trace over the orthogonal complement of this space. Using the methods developed for the one soliton case we can evaluate this as

$$
\operatorname{Tr}^{\prime}\left(e^{-i\left(\bar{k} S_{2} c S_{2}^{\dagger}+k S_{2} c^{\dagger} S_{2}^{\dagger}\right)}\right)=\operatorname{Tr}\left(e^{-i\left(\bar{k} c+k c^{\dagger}\right)}\right),
$$

which again leads to a constant background density, and

$$
\operatorname{tr}\left(e^{-i(D|0\rangle\langle 1|+\bar{D}| 1\rangle\langle 0|)}\right)=2 \cos (|D|),
$$

which gives us the perturbation part of the density

$$
\rho_{p}(\mathbf{x})=\int \frac{d^{2} \mathbf{k}}{(2 \pi)^{2}} 2 \cos (|D|) e^{i k_{a} x^{a}} .
$$

To do this integral we choose integration variables $k$ that simplify $|D|$ : we change coordinates from $\mathbf{k}$ to $\mathbf{q}$

$$
k=\frac{z}{2|z|}\left(\frac{q_{1}}{\lambda_{+}}+i \frac{q_{2}}{\lambda_{-}}\right)
$$

where we have defined

$$
\lambda_{ \pm}=\frac{|z|}{2}\left(\frac{1}{C} \pm C\right) .
$$

In these coordinates $D$ is simply

$$
|D|^{2}=q_{1}^{2}+q_{2}^{2} \text {. }
$$

Using the asymptotic expressions for $\mathrm{C}(3.46)$ we see that

$$
\begin{aligned}
& \lambda_{+}=\left\{\begin{array}{cc}
\frac{1}{2} & |z| \ll 1 \\
|z| & |z| \gg 1
\end{array}\right. \\
& \lambda_{-}=\left\{\begin{array}{cc}
\frac{1}{2} & |z| \ll 1 \\
0 & |z| \gg 1
\end{array}\right.
\end{aligned}
$$

The Jacobian is $\frac{1}{2 \theta \lambda_{+} \lambda_{-}}$so we have

$$
\rho_{p}(\mathbf{y})=\frac{1}{\theta \lambda_{+} \lambda_{-}} \int \frac{d^{2} \mathbf{q}}{(2 \pi)^{2}} \cos \left(\sqrt{q_{1}^{2}+q_{2}^{2}}\right) e^{i q_{a} y^{a}}
$$


where we have introduced a new variable $\mathbf{y}$ defined by $\mathbf{k} \cdot \mathbf{x} \equiv \mathbf{q} \cdot \mathbf{y}$, which implies

$$
\left(y_{1}, y_{2}\right)=\left(\frac{1}{4|z| \lambda_{+}}\left[x_{1}(z+\bar{z})+i x_{2}(\bar{z}-z)\right], \frac{1}{4|z| \lambda_{-}}\left[x_{2}(z+\bar{z})-i x_{1}(\bar{z}-z)\right]\right) .
$$

Note that a circle of radius 1 in the $\mathbf{y}$ coordinate system when transformed to $\mathbf{x}$ coordinates looks like an ellipse with the major axis oriented in the direction of $z$ and of length $\sqrt{2 \theta} \lambda_{+}$ and a minor axis of length $\sqrt{2 \theta} \lambda_{-}$. From (3.46) we also see that for small $|z|$ the ellipse becomes a circle of radius $\sqrt{\frac{\theta}{2}}$, whereas for large $|z|, \lambda_{-}$goes exponentially fast to zero so the ellipse is rapidly squeezed into a line between $\pm \sqrt{2 \theta} \lambda_{+} \rightarrow \pm \sqrt{2 \theta}|z|$.

We perform the angular integral and find

$$
\rho_{p}(\mathbf{y})=\frac{1}{\theta \lambda_{+} \lambda_{-}} \int \frac{d q}{2 \pi} q \cos q J_{0}(q y)=\frac{1}{\theta \lambda_{+} \lambda_{-}} \mathcal{R} e \int \frac{d q}{2 \pi} q e^{-i q} J_{0}(q y)
$$

where $y$ is the absolute value of the vector $\mathbf{y}$. This integral is divergent and needs to be regulated. This we do by adding a small real part to the argument in the exponential. Thus, with $a=i+\epsilon$ we have

$$
\rho_{p}(\mathbf{y})=\lim _{\epsilon \rightarrow 0} \frac{1}{\theta \lambda_{+} \lambda_{-}} \mathcal{R} e \int \frac{d q}{2 \pi} q e^{-a q} J_{0}(q y)=\lim _{\epsilon \rightarrow 0} \frac{1}{2 \pi \theta \lambda_{+} \lambda_{-}} \mathcal{R} e \frac{a}{\left(y^{2}+a^{2}\right)^{\frac{3}{2}}}
$$

which is completely well defined and smooth for all non-zero $\epsilon$. Letting $\epsilon \rightarrow 0$ the function is zero for $y>1$ and for $y<1$ it looks like $\rho_{p}=-\frac{1}{2 \pi \theta \lambda_{+} \lambda_{-}} \frac{1}{\left(1-y^{2}\right)^{\frac{3}{2}}}$. In figure 11 we see a cross section of the full density drawn in the $y$-plane where the border of the ellipse is at $|y|=1$ and for a small non-zero value of $\epsilon$. The background density is normalized to one.

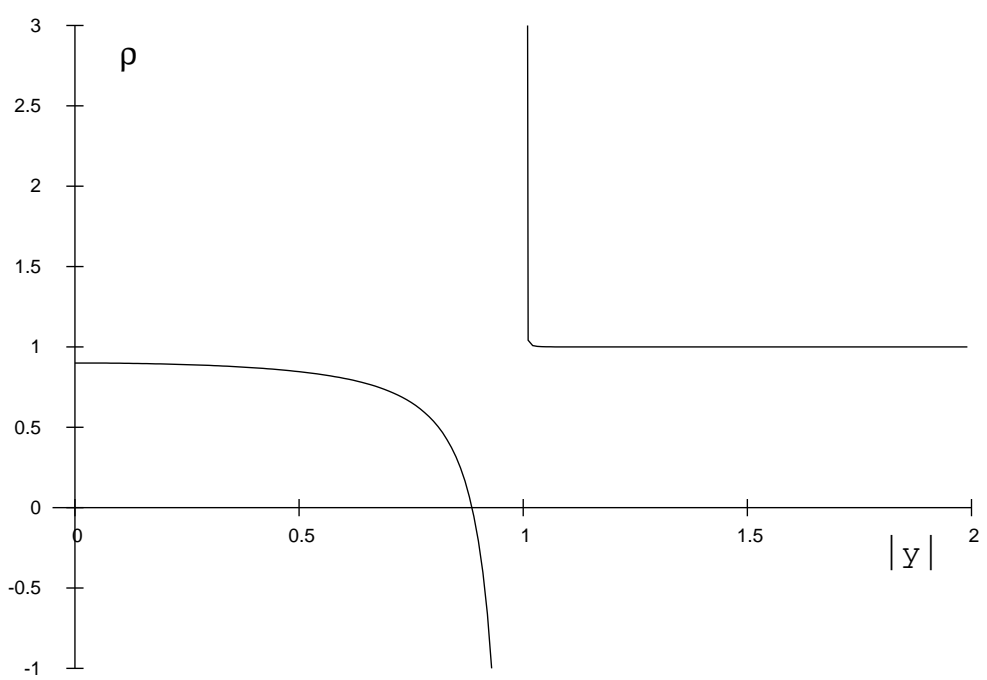

Figure 1: The full density

Notice that the full density inside the ellipse is always less than the background density. This means that we have taken particles from the inside of the ellipse and piled them up 
at the border of the ellipse. Notice also that there is always a small region on the inside of the border of the ellipse where the full density will be negative. This seems difficult to reconcile with the interpretation that the theory describes an electron gas since then there could not be less than zero electrons at any point.

As a final exercise we show how two one-soliton solution can be found in the $|z| \rightarrow \infty$ limit of the two-soliton solution. We want to show that

$$
\lim _{|z| \rightarrow \infty} \rho_{p}(\mathbf{x})=\delta^{(2)}(x-\sqrt{2 \theta} z)+\delta^{(2)}(x+\sqrt{2 \theta} z)
$$

To do this we have to use the regularized version of $\rho_{p}$ using the non-zero $\epsilon$ which we will take to zero only at the end of the calculation. Thus we would like to show that for any real function $f\left(x_{1}, x_{2}\right)$

$$
\begin{aligned}
f(\sqrt{2 \theta} z)+f(-\sqrt{2 \theta} z) & =\lim _{\epsilon \rightarrow 0} \lim _{|z| \rightarrow \infty} \int d^{2} \mathbf{x} f(\mathbf{x}) \rho_{p}(\mathbf{x}) \\
& =\lim _{\epsilon \rightarrow 0} \lim _{|z| \rightarrow \infty} \frac{1}{2 \pi \theta \lambda_{+} \lambda_{-}} \mathcal{R} e \int d^{2} \mathbf{x} f(\mathbf{x}) \frac{i}{\left(y(x)^{2}+(i+\epsilon)^{2}\right)^{\frac{3}{2}}}(
\end{aligned}
$$

Changing integration variables from $\mathbf{x}$ to $\mathbf{y}$, not forgetting the Jacobian, we get

$$
\lim _{\epsilon \rightarrow 0} \lim _{|z| \rightarrow \infty} \frac{1}{\pi} \mathcal{R} e \int d^{2} \mathbf{y} f\left(\sqrt{2 \theta} \lambda_{+} y_{1}, \sqrt{2 \theta} \lambda_{-} y_{2}\right) \frac{i}{\left(y^{2}+(i+\epsilon)^{2}\right)^{\frac{3}{2}}}
$$

But in the limit when $|z|$ goes to infinity, $\lambda_{-}$goes to zero exponentially fast. This means that the $y_{2}$ dependence of the function $f$ is scaled out and the integral becomes

$$
\begin{aligned}
\lim _{\epsilon \rightarrow 0} \lim _{|z| \rightarrow \infty} & \frac{1}{\pi} \mathcal{R} e \int d y_{1} f\left(\sqrt{2 \theta} \lambda_{+} y_{1}, 0\right) \int_{-\infty}^{+\infty} d y_{2} \frac{i}{\left(y^{2}+(i+\epsilon)^{2}\right)^{\frac{3}{2}}} \\
& =\lim _{\epsilon \rightarrow 0} \lim _{|z| \rightarrow \infty} \frac{1}{\pi} \mathcal{R} e \int d y_{1} f\left(\sqrt{2 \theta} \lambda_{+} y_{1}, 0\right) \frac{2 i}{y_{1}^{2}+(i+\epsilon)^{2}}
\end{aligned}
$$

Taking the real part and performing the $\epsilon \rightarrow 0$ limit we recognize a representation of the delta function

$$
\lim _{\epsilon \rightarrow 0} \mathcal{R} e \frac{2 i}{y_{1}^{2}-1+i \epsilon}=\lim _{\epsilon \rightarrow 0} \frac{2 \epsilon}{\left(y_{1}^{2}-1\right)^{2}+\epsilon^{2}}=2 \pi \delta\left(y_{1}^{2}-1\right)=\pi \delta\left(y_{1}-1\right)+\pi \delta\left(y_{1}+1\right)(3 .
$$

and substituting this back we get

$$
f\left(\sqrt{2 \theta} \lambda_{+}, 0\right)+f\left(-\sqrt{2 \theta} \lambda_{+}, 0\right)
$$

and since for large $|z|$ we have $\lambda_{+} \approx|z|$, this is what we wanted to show.

\subsubsection{The dynamics}

To study the dynamics on the two soliton moduli space one can try to make the moduli slowly time dependent and find the effective action in the moduli. Doing this we find

$$
i \pi \kappa \int d t\left(C^{2}-\frac{1}{C^{2}}\right)^{2}|z|^{2}(\bar{z} \dot{z}-z \dot{\bar{z}})
$$


or, using $z=r e^{i \phi}$,

$$
-2 \pi \kappa \int d t\left(C^{2}-\frac{1}{C^{2}}\right)^{2} r^{4} \dot{\phi}
$$

Notice that there is no time derivative of $r$ and that the function in front of $\dot{\phi}$ depends only on $r$. It can be written as $\left(4 \lambda_{+} \lambda_{-}\right)^{2}$ which is identical to the "angular momentum" of the two soliton configuration defined in [15]. Using the asymptotic expansions for $\mathrm{C}$ (3.46) we find that it goes as $\left(1-4 r^{4}\right)$ for small $r$ and for large $r$ it goes exponentially to zero.

The effective action is first order in time derivatives and thus comes in "Hamiltonian" form (with the Hamiltonian equal to zero). It defines a connection 1-form on the moduli space $\mathcal{A}=i\left(C^{2}-\frac{1}{C^{2}}\right)^{2}|z|^{2}(z d \bar{z}-\bar{z} d z)$ whose curvature 2-form is the symplectic form on the moduli space seen as a phase space. This may be taken as the starting point for quantization of the system but since the Hamiltonian is zero, there is no non-trivial time evolution. We have plotted the symplectic form in figure 2. At small $|z|$ it goes to zero as

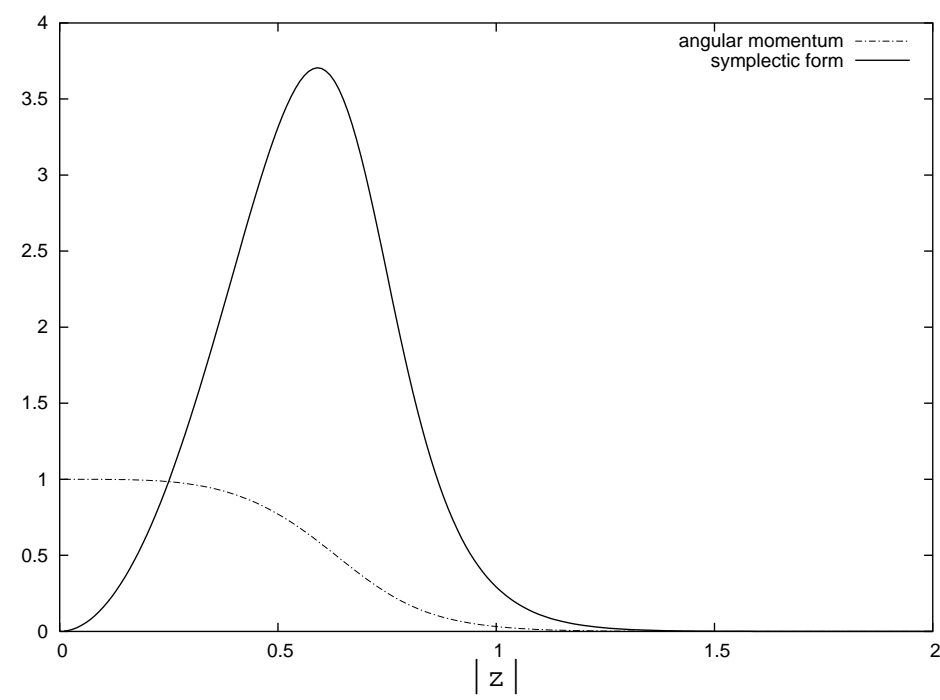

Figure 2: The angular momentum and the symplectic 2-form

$16|z|^{2}$ and at large $|z|$ it goes to zero exponentially ${ }^{4}$. In between (at $\left.|z| \approx 0.6\right)$ there is a maximum.

One could speculate that, as in [28], a potential for the moduli could be generated. This would then define a nontrivial Hamiltonian which would give non-trivial dynamics on the moduli space.

As found in [28, 29, 30] in the commutative Chern-Simons case, the symplectic form defined above equals the Kähler form of the metric on the moduli space of vortex solutions to the Maxwell-Higgs model. If this is true also in the non-commutative model, the symplectic form we just computed should have the properties of a Kähler metric. This seems to be true for small $|z|$ where the apparent conical singularity is removed after dividing by the $Z_{2}$ action that arises because the voritces are identical particles. At large $|z|$ however,

\footnotetext{
${ }^{4}$ Since the form degenerates at 0 and $\infty$, the phase space is singular at these points.
} 
the metric is zero, which seems to go against the intuition that the metric should be flat for well separated vortices (they should not interact at large distances). It also seems to disagree with explicit calculations of the metric in the Maxwell-Higgs case [31]. However, our result is similar to what we find in the relativistic case in the next section, where we again find a nontrivial metric between vortices even in the large $|z|$ limit. As in [30] one should be able to use our expression for the symplectic two form to calculate the phase space volume or rather the reduction of the phase space volume due to the presence of other vortices. From this one could find the statistics of the vortices.

There have been other attempts to find the dynamics of first order system of vortices, e.g., [33]. However, without additional assumptions the methods of [33] do not yield unique solutions, and we were not able to put them to fruitful use in our case.

\section{The relativistic scalar field}

The Lagrangian of a noncommutative Chern-Simons field interacting with a relativistic scalar field can be written [15] (using the objects defined in (3.4)) as

$$
\begin{aligned}
\mathrm{L}_{\mathrm{rel}}= & i \pi \kappa \operatorname{Tr}\left\{K^{\dagger} D_{t} K-K D_{t} K^{\dagger}\right\}-2 \pi \kappa \operatorname{Tr} A_{0}+ \\
& +2 \pi \theta \operatorname{Tr}\left\{D_{t} \phi\left(D_{t} \phi\right)^{\dagger}-\frac{1}{\theta}\left(D \phi(D \phi)^{\dagger}+\bar{D} \phi(\bar{D} \phi)^{\dagger}\right)-V\left(\phi \phi^{\dagger}\right)\right\}
\end{aligned}
$$

leading to the equations of motion

$$
\begin{gathered}
D_{t} D_{t} \phi-\frac{1}{\theta}\{D, \bar{D}\} \phi+V^{\prime}\left(\phi \phi^{\dagger}\right) \phi=0, \\
\kappa i D_{t} K=\left\{K, \phi \phi^{\dagger}\right\}-2 \phi c \phi^{\dagger}, \\
1-\left[K, K^{\dagger}\right]=i \frac{\theta}{\kappa}\left[D_{t} \phi \phi^{\dagger}-\phi\left(D_{t} \phi\right)^{\dagger}\right] .
\end{gathered}
$$

If the potential has the form

$$
V(\zeta)=\frac{1}{\kappa^{2}} \zeta\left(\zeta-u^{2}\right)^{2}
$$

where $u$ is a (real) constant, then the model has a Bogomolny bound and BPS equations:

$$
\begin{gathered}
\bar{D} \phi=0, \\
D_{t} \phi=\frac{i}{\kappa}\left(\phi \phi^{\dagger}-u^{2}\right) \phi, \\
1-\left[K, K^{\dagger}\right]=i \frac{\theta}{\kappa}\left[D_{t} \phi \phi^{\dagger}-\phi\left(D_{t} \phi\right)^{\dagger}\right] .
\end{gathered}
$$

There is also a second set of BPS equations in this model, with $D \phi=0$ and $D_{t} \phi=$ $-\frac{i}{\kappa}\left(\phi \phi^{\dagger}-u^{2}\right) \phi$, but these equations do not seem to have localized solutions as there are no normalizable eigenvectors of the creation operator $c^{\dagger}$. 
It is instructive to see how (4.2, 4.3,4.4) follow from (4.6,4.7,4.8). Substituting (4.7) into $(4.8)$ we get

$$
1-\left[K, K^{\dagger}\right]=-\frac{2 \theta}{\kappa^{2}}\left(\phi \phi^{\dagger}-u^{2}\right) \phi \phi^{\dagger} .
$$

From $\bar{D} \phi=0$ and the definitions of $D, \bar{D}$ it follows that

$$
-\frac{1}{\theta}\{D, \bar{D}\} \phi=\frac{1}{\theta}[D, \bar{D}] \phi=\frac{1}{\theta}\left(1-\left[K, K^{\dagger}\right]\right) \phi=-\frac{2}{\kappa^{2}}\left(\phi \phi^{\dagger}-u^{2}\right) \phi \phi^{\dagger} \phi .
$$

Computing the $D_{t}$ derivative of $(4.7)$, we get

$$
D_{t} D_{t} \phi=-\frac{i}{\kappa}\left(u^{2} D_{t} \phi-D_{t} \phi \phi^{\dagger} \phi-\phi\left(D_{t} \phi\right)^{\dagger} \phi-\phi \phi^{\dagger} D_{t} \phi\right) ;
$$

hence

$$
D_{t} D_{t} \phi=-\frac{1}{\kappa^{2}}\left(\phi \phi^{\dagger}-u^{2}\right)^{2} \phi
$$

and thus

$$
D_{t} D_{t} \phi-\frac{1}{\theta}\{D, \bar{D}\} \phi=-\frac{1}{\kappa^{2}}\left(\phi \phi^{\dagger}-u^{2}\right)\left(3 \phi \phi^{\dagger}-u^{2}\right) \phi \equiv-V^{\prime}\left(\phi \phi^{\dagger}\right) \phi .
$$

For $V$ of the form (4.5), this is (4.2).

To derive (4.3), we write

$$
0=D_{t}(\bar{D} \phi)=-\left(D_{t} K\right)^{\dagger} \phi-K^{\dagger} D_{t} \phi+D_{t} \phi c^{\dagger}
$$

which gives

$$
\phi^{\dagger} D_{t} K=c\left(D_{t} \phi\right)^{\dagger}-\left(D_{t} \phi\right)^{\dagger} K=-\frac{i}{\kappa}\left(c \phi^{\dagger} \phi \phi^{\dagger}-\phi^{\dagger} \phi \phi^{\dagger} K\right)
$$

multiplying with $\phi$ on the left, we get

$$
\phi \phi^{\dagger} i \kappa D_{t} K=\phi c \phi^{\dagger} \phi \phi^{\dagger}-\left(\phi \phi^{\dagger}\right)^{2} K \text {. }
$$

Noting that $\bar{D} \phi=0 \Rightarrow \phi \phi^{\dagger} K=\phi c \phi^{\dagger}$, this gives

$$
\phi \phi^{\dagger} i \kappa D_{t} K=\phi \phi^{\dagger}\left[K, \phi \phi^{\dagger}\right]=\phi \phi^{\dagger}\left(\left\{K, \phi \phi^{\dagger}\right\}-2 \phi c \phi^{\dagger}\right) .
$$

Here we see that, unlike in the non-relativistic case, a solution to the BPS equations imply a solution to the full equations of motion and not just to the time independent equations of motion. This is almost true. There could also exist BPS solutions where the operator

$$
i \kappa D_{t} K-\left\{K, \phi \phi^{\dagger}\right\}+2 \phi c \phi^{\dagger}
$$

is non-zero, but lies in the subspace orthogonal to the operator $\phi \phi^{\dagger}$. These "solutions" would then be solutions to the BPS equations only. 


\subsection{The one soliton case}

\subsubsection{The static solution}

The static, one soliton solution is given by the Ansatz [15]

$$
K=z|0\rangle\left\langle 0\left|+S_{1} c S_{1}^{\dagger}, \quad \phi=\lambda\right| 0\right\rangle\left\langle z\left|, \quad A_{0}=\frac{b}{\sqrt{2 \theta}}\right| 0\right\rangle\langle 0|
$$

where the parameters $\lambda$ and $b$ are real and are determined in terms of the parameters $\kappa, \theta, u$

in the Lagrangian. It follows from the equations of motion $4.2,4.3,4.4$ ) that $b=\frac{\kappa}{\sqrt{2 \theta} \lambda^{2}}$ and

$$
\frac{\lambda^{2}}{u^{2}}=\frac{1}{2}\left(1 \pm \sqrt{1-\frac{2 \kappa^{2}}{\theta u^{4}}}\right) \quad \text { or } \quad \frac{\lambda^{2}}{u^{2}}=\frac{1}{6}\left(1 \pm \sqrt{1+\frac{6 \kappa^{2}}{\theta u^{4}}}\right) \text {. }
$$

The first two values correspond to configurations that are also solutions in the Bogomol'nyi limit. Note that they exist only for $\theta>2 \kappa^{2} / u^{4}$, whereas the remaining two solutions are well defined for any nonvanishing noncommutativity parameter.

The $K$ part of the solution has the same form as in the nonrelativistic case and the shapes of relativistic and nonrelativistic solitons coincide.

\subsubsection{More stationary solutions}

Encouraged by the results from the nonrelativistic model, one may try to find stationary solutions with the same charge density as the BPS solutions. In this case however, one would have to use noncommutative Lorenz boosts [35] instead of a Galilean boosts to make the solitons move. We have not worked out the details of this but we feel confident that the solutions can be found.

\subsection{The two soliton case}

\subsubsection{The solution}

As in the nonrelativistic case the two soliton solution can be written in the form [15]

$$
\begin{aligned}
\phi & =\lambda(\sin \alpha|0\rangle\langle+|+\cos \alpha| 1\rangle\langle-|), \\
K & =z\left(C|0\rangle\left\langle 1\left|+\frac{1}{C}\right| 1\right\rangle\langle 0|\right)+S_{2} c S_{2}^{\dagger},
\end{aligned}
$$

and the BPS equations (4.6,4.7,4.8) give

$$
\begin{array}{r}
1-|z|^{2}\left(C^{2}-C^{-2}\right)=\frac{2 \theta \lambda^{2}}{\kappa^{2}} \sin ^{2} \alpha\left(u^{2}-\lambda^{2} \sin ^{2} \alpha\right) \\
1+|z|^{2}\left(C^{2}-C^{-2}\right)=\frac{2 \theta \lambda^{2}}{\kappa^{2}} \cos ^{2} \alpha\left(u^{2}-\lambda^{2} \cos ^{2} \alpha\right), \\
\cot ^{2} \alpha=C^{2} \tanh |z|^{2} .
\end{array}
$$

Unlike the nonrelativistic case, it is not possible to solve (4.13) in a closed form. It is however straightforward to find approximate solutions for small and large soliton separations. 
We find

$$
\begin{aligned}
C & =|z|+\left(\frac{\theta u^{2} \lambda_{0}^{2}}{\kappa^{2}}-\frac{1}{2}\right)|z|^{5}+\mathcal{O}\left(|z|^{9}\right), \\
\cot \alpha & =|z|^{2}+\left(\frac{\theta u^{2} \lambda_{0}^{2}}{\kappa^{2}}-\frac{1}{3}\right)|z|^{6}+\mathcal{O}\left(|z|^{10}\right), \\
\lambda^{2} & =\lambda_{0}^{2}-\frac{2 \lambda_{0}^{4}}{u^{2}-2 \lambda_{0}^{2}}|z|^{4}+\mathcal{O}\left(|z|^{8}\right), \\
\lambda_{0}^{2} & =\frac{u^{2}}{2}\left(1 \pm \sqrt{1-\frac{4 \kappa^{2}}{\theta u^{4}}}\right),
\end{aligned}
$$

for $|z| \ll 1$ and

$$
\begin{aligned}
C & =1-\frac{\theta \lambda_{\infty}^{2}\left(u^{2}-\lambda_{\infty}^{2}\right)}{4 \kappa^{2}|z|^{2}-\theta \lambda_{\infty}^{2}\left(2 u^{2}-\lambda_{\infty}^{2}\right)} \mathrm{e}^{-2|z|^{2}}+\mathcal{O}\left(\mathrm{e}^{-4|z|^{2}}\right), \\
\cot \alpha & =1-\frac{4 \kappa^{2}|z|^{2} \mid}{4 \kappa^{2}|z|^{2}-\theta \lambda_{\infty}^{2}\left(u^{2}-\lambda_{\infty}^{2}\right)} \mathrm{e}^{-2|z|^{2}}+\mathcal{O}\left(\mathrm{e}^{-4|z|^{2}}\right), \\
\lambda^{2} & =\lambda_{\infty}^{2}+\mathcal{O}\left(\mathrm{e}^{-4|z|^{2}}\right) \\
\lambda_{\infty}^{2} & =u^{2}\left(1 \pm \sqrt{1-\frac{2 \kappa^{2}}{\theta u^{4}}}\right)
\end{aligned}
$$

for $|z| \gg 1$.

Note that - modulo different dependence of the coefficients on $z$ - the form of $K$ in relativistic and nonrelativistic cases coincide and the leading terms in the $C$ expansions for small and large $|z|$ are the same. Therefore the analysis of the shape of the two soliton solution performed in section 3.2 .2 can be repeated for the relativistic solution virtually without any changes.

\subsubsection{The dynamics}

To understand the dynamics of slowly moving solitons we use Manton's method of moduli space approximation [36, 37] (see also [9, 10]). The effective action, obtained from (4.1) by inserting the ansatz (4.12) with time dependent moduli $z$ has the form

$$
S_{\text {eff }}=2 \pi \theta \int d t\left[g_{z z} \dot{z}^{2}+g_{z \bar{z}} \dot{z} \dot{\bar{z}}+g_{\bar{z} \bar{z}} \dot{\bar{z}}^{2}+i \mathcal{A}(z \dot{\bar{z}}-\dot{z} \bar{z})\right]
$$

with

$$
\begin{aligned}
g_{z z}= & \left(\partial_{z} \lambda\right)^{2}+\lambda^{2}\left[\left(\partial_{z} \alpha\right)^{2}-\frac{\bar{z}^{2}}{4}\left(\tanh ^{2}|z|^{2} \sin ^{2} \alpha+\operatorname{coth}^{2}|z|^{2} \cos ^{2} \alpha\right)\right], \\
g_{z \bar{z}}= & 2\left|\partial_{z} \lambda\right|^{2}+2 \lambda^{2}\left[\left|\partial_{z} \alpha\right|^{2}-\frac{|z|^{2}}{4}\left(\tanh ^{2}|z|^{2} \sin ^{2} \alpha+\operatorname{coth}^{2}|z|^{2} \cos ^{2} \alpha\right)\right] \\
& +\lambda^{2}\left[|z|^{2}+\tanh |z|^{2} \sin ^{2} \alpha+\operatorname{coth}|z|^{2} \cos ^{2} \alpha\right], \\
g_{\bar{z} \bar{z}}= & \overline{g_{z z}}, \\
\mathcal{A}= & \frac{\kappa}{\theta \sinh 2|z|^{2}}\left[\cosh 2|z|^{2}+|z|^{2}\left(C^{2}-\frac{1}{C^{2}}\right)-\frac{1}{2} \sinh 2|z|^{2}\left(C^{2}+\frac{1}{C^{2}}\right)\right] .
\end{aligned}
$$


Using (4.13) it is straightforward to express $\partial_{z} \lambda$ and $\partial_{z} \alpha$ in terms of $\lambda, \alpha$ and $C$, but the result is complicated and not particularly illuminating and we refrain from giving it here. We also not analyze the dynamics that follows from (4.22) in detail and discuss only the limiting cases of small and large $|z|$.

For $|z| \ll 1$ the effective action takes the form

$$
S_{\mathrm{eff}}=2 \pi \theta \lambda_{0}^{2} \int d t r^{2}\left[3\left(2 \dot{r}^{2}+r^{2} \dot{\varphi}^{2}\right)+\frac{2 u^{2}}{\kappa} r^{2} \dot{\varphi}\right]+\mathcal{O}\left(r^{6} \dot{r}^{2}, r^{8}\right)
$$

where $z=r \mathrm{e}^{i \varphi}$ and we have dropped a total derivative term. In the coordinates

$$
R=r^{2}, \quad \vartheta=\sqrt{2} \varphi,
$$

this action reads

$$
S_{\text {eff }}=3 \pi \theta \lambda_{0}^{2} \int d t\left(\dot{R}^{2}+R^{2} \dot{\vartheta}^{2}+\frac{2 \sqrt{2} u^{2}}{3 \kappa} R^{2} \dot{\vartheta}\right)
$$

and leads to the equation of motion

$$
\begin{aligned}
& \ddot{R}-R\left(\dot{\vartheta}+\frac{2 \sqrt{2} u^{2}}{3 \kappa}\right) \dot{\vartheta}=0, \\
& \frac{d}{d t}\left[R^{2}\left(\dot{\vartheta}+\frac{\sqrt{2} u^{2}}{3 \kappa}\right)\right]=0 \Rightarrow \dot{\vartheta}=\frac{c_{1}}{R^{2}}-\frac{\sqrt{2} u^{2}}{3 \kappa},
\end{aligned}
$$

where $c_{1}$ is an integration constant.

The simplest solution of (4.26) correspond to solitons circulating around each other with fixed angular velocity,

$$
R=\text { const }, \quad \dot{\vartheta}=-\frac{2 \sqrt{2} u^{2}}{3 \kappa}, \quad \Leftrightarrow \quad r=\text { const }, \quad \dot{\varphi}=-\frac{2 u^{2}}{3 \kappa} .
$$

Another simple solution is obtained for $c_{1}=0$,

$$
r^{4}=c_{2}^{2} \sin ^{2}\left[\frac{\sqrt{2} u^{2}}{3 \kappa}\left(t-t_{0}\right)\right], \quad \dot{\varphi}=-\frac{u^{2}}{3 \kappa},
$$

and describes head-on scattering of the solitons.

In the general case of $c_{1} \neq 0$ it is possible (neglecting a term $\frac{2 u^{4}}{9 \kappa^{2}} r^{4}$ in comparison with $c_{1}^{2} r^{-4}$, which is consistent with the approximation made in deriving (4.24)) to write down the solution in the form

$$
r^{4}=c_{3}^{2}\left(t-t_{0}\right)^{2}+\frac{c_{1}^{2}}{c_{3}^{2}}, \quad \dot{\varphi}=-\frac{u^{2}}{3 \kappa}+\frac{1}{\sqrt{2}} \frac{c_{1} c_{3}^{2}}{c_{1}^{2}+c_{3}^{4}\left(t-t_{0}\right)^{2}} .
$$

In the region $|z| \gg 1$ one gets

$$
S_{\text {eff }}=2 \pi \theta \lambda_{\infty}^{2} \int d t r^{2}\left(\dot{r}^{2}+2 r^{2} \dot{\varphi}^{2}\right)+\mathcal{O}\left(\mathrm{e}^{-2|z|^{2}}\right)
$$


In the variables

$$
R=r^{2}, \quad \vartheta=2 \sqrt{2} \varphi
$$

(4.27) takes a form of a free particle action. Consequently the general solution of the equations of motion that follow from (4.27) can be presented in the form

$$
r^{2} \mathrm{e}^{i 2 \sqrt{2} \varphi}=\zeta_{0} t+\zeta_{1}
$$

with (complex) constants $\zeta_{0}$ and $\zeta_{1}$.

We thus see that the naive moduli space has conical singularities in both the short and long distance limit. This is so even for identical particles where we have to quotient the moduli space by a $Z_{2}$ action, which for the original GMS solitons removed the apparent conical singularity at the center of the moduli space [10, 38]. However, the result agrees with what we found in the non-relativistic case where the phase space is singular both in the long and short distance limit.

\section{Conclusions}

We have studied one and two soliton solutions in noncommutative Chern-Simons theory coupled to nonrelativistic and relativistic scalar fields. We studied the shape of the solitons using the exact Seiberg-Witten map. We have found new stationary solutions with timeindependent charge and energy densities. The most general state could be written as a certain superposition of basis states. We have also integrated the infinitesimal Galilean transformations of [27], and used them to generate moving solitons.

For the two soliton solutions we tried to study the dynamics using the moduli space approximation. The direct approach is possible only in the relativistic case because in the nonrelativistic case the action is linear in time derivatives. In the relativistic case, we found explicit expressions for the metric in the limits where the solitons are far apart and when they are close. In these regimes the metric is flat with a conical singularity at the center. However, the conical singularity is different in the two limits. This makes the interactions "long range" and the solitons affect each other at large distances. This awkward long range interaction could be avoided, but only at the price of an equally awkward redefinition of the physical variables. In the nonrelativistic case, we could not find a metric, but found a symplectic form that has singularities that resemble the singularities of the metric in the relativistic case.

Noncommutative theories are known to link UV and IR behavior; the long range interaction that we find could be related to this, and could be probed by studying the theory on a cylinder or torus. The physical meaning of the model should be elucidated by coupling to a physical external electromagnetic field.

The new types of solutions require further study. We should investigate the meaning of the gauge-invariant observables that distinguish the basis states with quantized energy from the general mixed states. We should also determine if such solutions arise in other theories, e.g., the noncommutative Maxwell-Higgs system. 


\section{Note added:}

An earlier version of this paper found only a special subfamily of the solutions that we discovered here, and were led (falsely) to conclude that no Galilean transformations are possible, and that moving solitons travel with quantized velocities. We thank P. A. Horvathy, L. Martina and P. C. Stichel for directing our attention to [27] and enlightening email exchange on the subject. We have been informed by the authors that they have also written a new paper on related topics [39]. We also thank D. Bak for useful communications.

\section{Acknowledgments:}

We are grateful to Hans Hansson and Anders Karlhede for illuminating discussions. We thank our respective institutions for generously hosting the other members of the collaboration at various times during this project. LH and UL are grateful for the stimulating atmosphere at the Simons Workshop in Mathematics and Physics at the C.N. Yang Institute for Theoretical Physics, Stony Brook, where this work was completed. The work of LH was supported by the EC IHP network HPRN-CT-1999-000161. UL acknowledges support in part by EU contract HPNR-CT-2000-0122 and by VR grant 650-1998368. The work of MR was supported in part by NSF Grant No. PHY-0098527 and Supplement for International Cooperation with Central and Eastern Euorpe PHY 0300634. The work of RvU was supported by the Czech Ministry of Education under Contracts No. 143100006 , GAČR 201/03/0512, and ME649.

\section{References}

[1] N. Seiberg and E. Witten, String theory and noncommutative geometry, JHEP 9909, 032 (1999) [arXiv:hep-th/9908142].

[2] J. A. Harvey, Komaba lectures on noncommutative solitons and D-branes, arXiv:hep-th/0102076.

[3] M. R. Douglas and N. A. Nekrasov, Noncommutative field theory, Rev. Mod. Phys. 73, 977 (2001) [arXiv:hep-th/0106048].

[4] R. J. Szabo, Quantum field theory on noncommutative spaces, Phys. Rept. 378, 207 (2003) [arXiv:hep-th/0109162].

[5] R. Gopakumar, S. Minwalla and A. Strominger, Noncommutative solitons, JHEP 0005, 020 (2000) [arXiv:hep-th/0003160].

[6] V. Schomerus, D-branes and deformation quantization, JHEP 9906, 030 (1999) [arXiv:hep-th/9903205].

[7] L. Susskind, The quantum Hall fluid and noncommutative Chern Simons theory, arXiv:hep-th/0101029.

[8] A. P. Polychronakos, Quantum Hall states as matrix Chern-Simons theory, JHEP 0104, 011 (2001) [arXiv:hep-th/0103013].

[9] U. Lindström, M. Roček and R. von Unge, Non-commutative soliton scattering, JHEP 0012 , 004 (2000) [arXiv:hep-th/0008108]. 
[10] L. Hadasz, U. Lindström, M. Roček and R. von Unge, Noncommutative multisolitons: Moduli spaces, quantization, finite Theta effects and stability, JHEP 0106, 040 (2001) [arXiv:hep-th/0104017].

[11] S. Hellerman and L. Susskind, Realizing the quantum Hall system in string theory, arXiv:hep-th/0107200.

[12] B. Freivogel, L. Susskind and N. Toumbas, A two fluid description of the quantum Hall soliton, arXiv:hep-th/0108076.

[13] T. H. Hansson and A. Karlhede, Charges and Currents in the Noncommutative Chern-Simons Theory of the QHE, arXiv:cond-mat/0109413.

[14] T. H. Hansson, J. Kailasvuori and A. Karlhede, Charge and Current in the Quantum Hall Matrix Model, arXiv:cond-mat:0304271.

[15] D. Bak, S. K. Kim, K. S. Soh and J. H. Yee, Noncommutative Chern-Simons solitons, Phys. Rev. D 64, 025018 (2001) [arXiv:hep-th/0102137].

[16] G. S. Lozano, E. F. Moreno and F. A. Schaposnik, Self-dual Chern-Simons solitons in noncommutative space, JHEP 0102, 036 (2001) [arXiv:hep-th/0012266].

[17] R. Jackiw and S. Y. Pi, Classical And Quantal Nonrelativistic Chern-Simons Theory, Phys. Rev. D 42, 3500 (1990) [Erratum-ibid. D 48, 3929 (1993)].

[18] R. Jackiw, S. Y. Pi and E. J. Weinberg, Topological And Nontopological Solitons In Relativistic And Nonrelativistic Chern-Simons Theory, MIT-CTP-1857 Presented at PASCOS '90, Boston, MA, Mar 27-31, 1990

[19] Y. Okawa and H. Ooguri, An exact solution to Seiberg-Witten equation of noncommutative gauge theory, Phys. Rev. D 64 (2001) 046009 arXiv:hep-th/0104036.

[20] S. Mukhi and N. V. Suryanarayana, Gauge-invariant couplings of noncommutative branes to Ramond-Ramond backgrounds, JHEP 0105 (2001) 023 arXiv:hep-th/0104045.

[21] H. Liu and J. Michelson, Ramond-Ramond couplings of noncommutative D-branes, Phys. Lett. B 518 (2001) 143 arXiv:hep-th/0104139.

[22] H. Liu, *-Trek II: *n operations, open Wilson lines and the Seiberg-Witten map, Nucl. Phys. B 614 (2001) 305 arXiv:hep-th/0011125.

[23] J. H. Brodie, L. Susskind and N. Toumbas, How Bob Laughlin tamed the giant graviton from Taub-NUT space, JHEP 0102 (2001) 003 [arXiv:hep-th/0010105].

[24] A. P. Polychronakos, Noncommutative Chern-Simons terms and the noncommutative vacuum, JHEP 0011 (2000) 008 [arXiv:hep-th/0010264].

[25] J. Klusoñ, Matrix model and noncommutative Chern-Simons theory, Phys. Lett. B 505 (2001) 243 [arXiv:hep-th/0012184].

[26] O. Bergman, Y. Okawa and J. H. Brodie, The stringy quantum Hall fluid, JHEP 0111 (2001) 019 arXiv:hep-th/0107178.

[27] P. A. Horvathy, L. Martina and P. C. Stichel, Galilean noncommutative gauge theory: Symmetries and vortices, Nucl. Phys. B 673 (2003) 301 [arXiv:hep-th/0306228].

[28] N. S. Manton, First order vortex dynamics, Annals Phys. 256 (1997) 114 [arXiv:hep-th/9701027]. 
[29] N. M. Romao, Quantum Chern-Simons vortices on a sphere, J. Math. Phys. 42 (2001) 3445 [arXiv:hep-th/0010277].

[30] T.H. Hansson, S.B. Isakov, J.M. Leinaas, and U. Lindström, Classical phase space and statistical mechanics of identical particles Phys. Rev. E 63 (2001) 026102 [arXiv:quant-ph/0003121].

[31] D. Tong, The moduli space of noncommutative vortices, arXiv:hep-th/0210010.

[32] D. Tong, A Quantum Hall Fluid of Vortices, arXiv:hep-th/0306266.

[33] J. Dziarmaga, More on scattering of Chern-Simons vortices, Phys. Rev. D 51 (1995) 7052 [arXiv:hep-th/9412180].

[34] L. Landau, E .Lifshitz Quantum Mechanics. nonrelativistic theory. Pergamon Press 1958. Appendix §a.

[35] D. Bak and K. M. Lee, Elongation of moving noncommutative solitons, Phys. Lett. B 495, 231 (2000) [arXiv:hep-th/0007107].

[36] N. S. Manton, A Remark On The Scattering Of BPS Monopoles, Phys. Lett. B 110, 54 (1982).

[37] T. M. Samols, Vortex Scattering, Commun. Math. Phys. 145, 149 (1992).

[38] R. Gopakumar, M. Headrick and M. Spradlin, On noncommutative multi-solitons, Commun. Math. Phys. 233 (2003) 355 [arXiv:hep-th/0103256].

[39] P. A. Horvathy, L. Martina and P. C. Stichel, to appear. [arXiv:hep-th/0311157]. 